\title{
Qualification of Barrel Pixel Detector Modules for the Phase 1 Upgrade of the CMS Vertex Detector
}

\author{
Simon Kudella* on behalf of the CMS Collaboration \\ Karlsruhe Institute of Technology \\ Institut für Experimentelle Kernphysik \\ Hermann-von-Helmholtz-Platz 1 \\ D-76344 Eggenstein-Leopoldshafen, Germany \\ E-mail: simon.kudella@cern.ch
}

To withstand the higher particle rates of LHC Runs 2 and 3, with expected luminosities of up to $2 \times 10^{34} \mathrm{~cm}^{-2} \mathrm{~s}^{-1}$, the current CMS pixel detector at the LHC will be replaced as part of the CMS Phase I Upgrade during the extended winter shutdown in 2016/17. The new pixel detector features a new geometry with one additional detector layer in the barrel region (BPIX) and one pair of additional disks in the forward region (FPIX), new digital readout chips as well as a new $\mathrm{CO}_{2}$-based cooling system for both the barrel and forward region. The BPIX detector module production is summarized, with special focus on the different stages of quality assurance. The quality tests as well as the calibrations which all produced modules undergo in a temperature and humidity controlled environment are described. Exemplarily, the KIT/Aachen production line and its subprocesses are presented together with their quality and yields.

The 25th International Workshop on Vertex Detectors

September 26-30, 2016

La Biodola, Isola d'Elba, ITALY

*Speaker. 

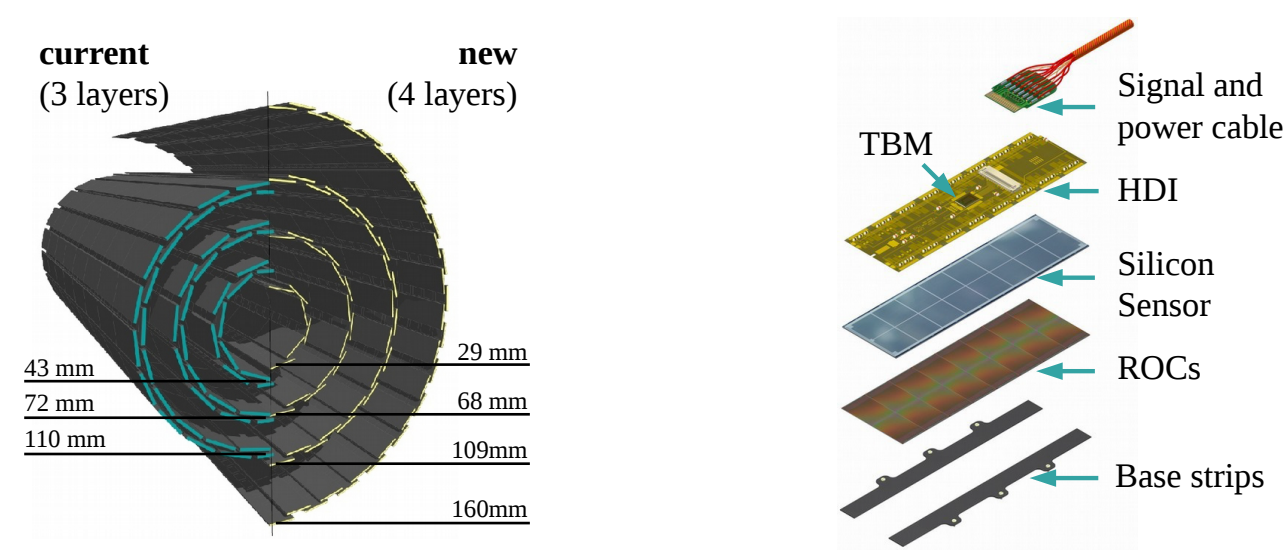

Figure 1: The figure on the left shows a comparison between the geometry of the current CMS pixel detector and the one after the Phase I Upgrade. An exploded view of a CMS Phase I BPIX module for layers 2-4 is shown on the right [1].

\section{CMS Phase I Upgrade}

In the upcoming years, the instantaneous luminosity of the Large Hadron Collider (LHC) at CERN will increase to twice the design luminosity $\left(2 \times 10^{34} \mathrm{~cm}^{-2} \mathrm{~s}^{-1}\right)$. To deal with the increase in particle rate and the radiation damage, CMS decided to completely replace its current pixel detector with an upgraded version. An additional fourth layer in the barrel region (BPIX) and additional third disks in the endcap regions (FPIX) increase the tracking redundancy, while the innermost layer gets moved closer to the interaction point, increasing the tracking precision. Figure 1 shows a comparison between the geometries of the current and the upgraded CMS Barrel Pixel Detector. All new modules will be equipped with new digital Readout Chips (ROCs), with less cross talk between neighbouring pixels and increased data buffers. At the same time, a more powerful twophase $\mathrm{CO}_{2}$-cooling system and a carbon fiber support structure reduce the total material budget and multiple scattering of particles. A total of 1184 good BPIX modules and 672 good FPIX modules are needed to equip the whole new detector. To provide such a large number of modules, the production was spread among five BPIX production centers and two FPIX production centers [1]. In the following sections, the "KIT/Aachen BPIX" production line will be presented exemplarily.

The module is the smallest subunit of the CMS pixel detector. A BPIX module is shown in Fig. 1. The central part of the module is its passive $\mathrm{n}^{+}$-in-n silicon sensor with 66560 pixel cells of $100 \times 150 \mu \mathrm{m}^{2}$ each, where ionizing particles create a charge signal. Each of these pixel cells is connected to a corresponding Pixel Unit Cell (PUC) housed in one of 16 ROCs. The interconnection between sensor pixel cells and PUCs is established with the bump bonding interconnection technology. The communication between ROCs and the CMS readout electronics is managed by the Token Bit Manager (TBM) on a High Density Interconnect (HDI) flex print, glued to the sensor. Electrical connections between ROCs, TBM and HDI are established by wire bonds. The connection between a module and the outside is provided by a twisted pair cable, distributing signals and power. For additional mechanical stability, handling, and mounting of the module, two SiN base strips are glued to the ROCs [1]. 

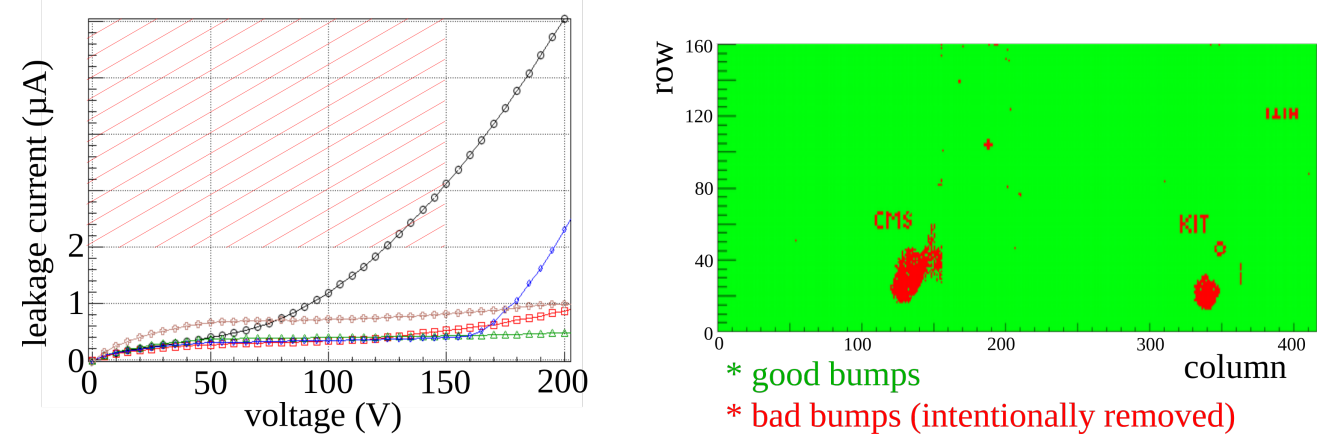

Figure 2: On the left, the IV selection criteria for the BPIX pixel sensors are visualized [2]. The right figure shows the output of the KIT bump bonding test, performed on a modified bare module using the KIT bare module probe station. The test returns a map of good and bad bump bond connections and their positions on the bare module [3].

\section{KIT/Aachen BPIX Module Production Line}

In the KIT/Aachen consortium, the BPIX module production line was seperated into the module assembly at the Karlsruhe Institute of Technology (KIT) and the module qualification at RWTH Aachen University. In order to maximize yield and module quality, KIT/Aachen decided to screen failures as early as possible by introducing several screenings and tests during the assembly. This allowed a direct quality feedback with very short return cycles. By seperating the bump bonding process into an external bumping process at RTI International and an internal flip-chip bonding process at KIT, the costs were reduced while providing a direct quality feedback. In the following sections, the process yields are divided into three quality classes: Grade A - very good for detector installation, Grade B - good with minor defects, but still good for detector installation, Grade C - bad.

\subsection{Component Preprocessing and Component Selection}

All KIT sensors were produced by CiS and were further processed by PacTech who deposited the Under Bump Metallization (UBM) and diced the sensor tiles. When delivered to KIT, all sensors underwent an electrical test in which they had to fulfill several requirements concerning the leakage current $\left(I_{\text {leak }}\right): I_{\text {leak }}(150 \mathrm{~V})<2 \mu \mathrm{A}, \frac{I_{\text {leak }}(150 \mathrm{~V})}{I_{\text {leak }}(100 \mathrm{~V})}<2$ (Fig. 2). In addition, an optical inspection checked for UBM defects, bad dicing or visible failures in the microelectronic structures. To check for defects inside the sensor bulk, an additional test was introduced at KIT, where a single ROC was bump bonded to the sensor and a second IV test through the sensor bulk was performed. In this way it was possible to sort out sensors with so far undiscovered bulk defects. If the sensor passed, the bump bonding of the other ROCs continued. All ROCs were produced by IBM in a $250 \mathrm{~nm}$ CMOS process and were electrically tested at Paul Scherrer Institute (PSI) before further processing. For the KIT/Aachen production line, the ROCs were getting equipped with bumps of eutectic $\mathrm{SnPb}(\varnothing=40 \mu \mathrm{m})$, thinned and diced by RTI [4]. To protect the bumps from mechanical damage as well as from oxidation during delivery, the ROCs were coverd by a thick photoresist layer. At KIT, this photoresist was removed by a dedicated cleaning process. Afterwards, an 
optical inspection was performed to identify defects caused by cleaning, bumping or dicing [5, 6]. The KIT selection criteria for the ROCs were: no defects in the electrical readout of the PUCs and in the data transfer of the ROC during the electrical test, $<5$ damaged bumps, as well as a maximum digital current $I_{\text {dig }} \leq 65 \mathrm{~mA}$ drawn by a ROC [5].

The TBMs were produced by IBM and the HDIs were produced by Hightec MC AG. After an optical inspection, the TBM was glued to the HDI and the electrical connection was established by aluminium wedge wire bonding. The electrical behaviour of the HDI-TBM assembly was tested using an oscilloscope and a needle card to contact the HDI. The SiN base strips were produced by the KYOCERA Corperation and laser cut into shape. All base strips were sorted by thickness and were only used in pairs with a thickness difference of $<5 \mu \mathrm{m}$ in order to achieve good planar and homogeneous base strip gluing. Considering these selection criteria, the following yields for good material emerged during the production: sensor yield $=81 \%$, ROC yield $=87 \%$, HDI \& TBM yield $=86 \%$, base strip yield $=90 \%$.

\subsection{Flip-Chip Bonding}

At KIT, the flip-chip bump bonding of ROCSs to sensors into so called "bare modules" was performed in a semi-automated process, using a FINEPLACER ${ }^{\circledR}$ femto flip-chip bonder machine and a dedicated vacuum reflow oven. The process was divided into several subprocesses: bonding, bare module testing, reworking if necessary, and reflow. All 16 ROCs were sequentially flip-chip bonded onto the silicon sensor, only interrupted by the bare module pretest as mentioned above. The bonding of a single ROC (tacking) to the sensor was performed by thermocompression. During this operation, the ROCs and the sensor were kept under $\mathrm{N}_{2}$-atmosphere to avoid any oxidation of the $\mathrm{SnPb}$ bumps. After bonding all ROCs to the sensor, the bare modules were tested on a probe station using a needle card to contact the ROCs. The tests performed on the bare modules included: IV measurement of the sensor, electrical test of the ROC readout chain and identification of bump bonding failures. To do so, the PUCs capacitively induce a charge signal into the sensor pixel cell. If the bump bonding connection is good, the signal can be read out by the PUC. An exemplary outcome of the bare bump bonding test is shown in Fig. 2. If the testing of the bare module shows a bad ROC, it can be reworked and replaced with a new one. A bare module failing in the IV measurement cannot be reworked. Finally, the strength of the bump bonding connection as well as the alignment and planarity of the assembly was improved by a vacuum reflow. The whole process showed a placement precision of $\sigma_{x y}<1 \mu \mathrm{m}$ and a planarity of $\theta_{\text {missplan }}<0.3 \mathrm{mrad}$ [5]. About $19 \%$ of the bare modules underwent reworking, which was successful in $90 \%$ of the cases. The total yield of the flip-chip bonding process was: Grade $\mathrm{A}=92 \%$, Grade $\mathrm{B}=3 \%$, Grade $\mathrm{C}=5 \%$.

\subsection{Full Module Assembly and Wire Bonding}

The mechanical connection between the components was established by gluing the bare module to the base strips and the HDI on top of the sensor, using Araldite ${ }^{\circledR} 2011$. To provide planar and homogeneous gluings with an alignment precision $<50 \mu \mathrm{m}$ and gluing layer thicknesses of $<10 \mu \mathrm{m}$, custom designed $z$-stages and glue stamps were used for all gluing steps. Since Araldite ${ }^{\circledR} 2011$ has a cure-out time of about 24 hours, this process was parallelized in four assembly lines allowing the assembly of four full modules a day. The electrical connection between HDI and ROCs as well as between HDI and sensor was established by aluminium wedge wire 


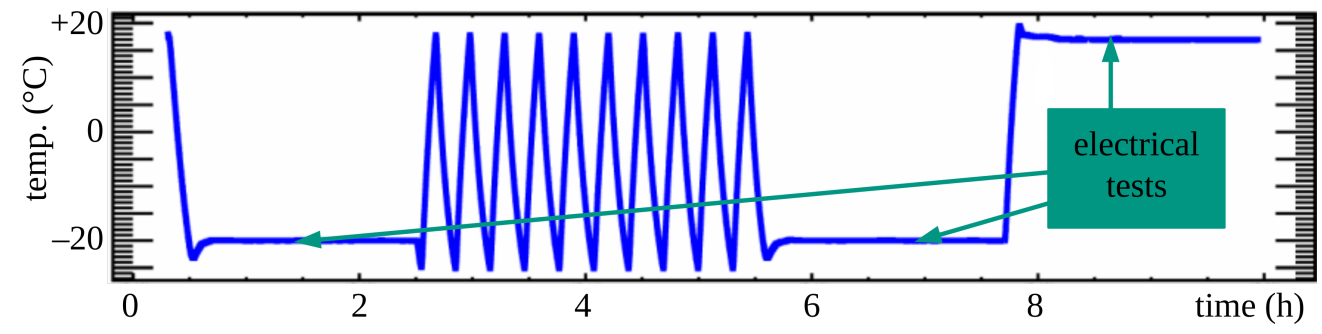

Figure 3: The test procedure for the thermal cycling and electrical tests [8].

bonding. Before sending the modules to RWTH Aachen University, another electrical test as well as an X-ray test were performed at KIT to give quick quality feedback on the assembly processes. These tests showed the following yields for the assembly line: Grade $\mathrm{A}=58 \%$, Grade $\mathrm{B}=36 \%$, Grade $\mathrm{C}=6 \%$.

The large percentage of Grade B modules is caused by the leakage current of the sensors not following the equation expected to describe the dependence of the leakage current $I$ from the temperature $T$ :

$$
I(T) \propto T^{2} \exp \left(\frac{-1.21 \mathrm{eV}}{2 \mathrm{k}_{\mathrm{B}} T}\right)
$$

(as determined in [7], with $\mathrm{k}_{\mathrm{B}}$ being the Boltzmann constant). Deviations from equation 2.1 might indicate small sensor defects causing non-thermally induced leakage currents within the sensor structure. Still, this behaviour is not critical for operation at the LHC, since it will be negligible compared to the leakage current contribution from radiation-induced bulk defects, after a few weeks of operation.

\subsection{Thermal Cycling and Electrical Test}

At RWTH Aachen University, all modules were tested and graded. The first test was focused on long-term reliability and module behaviour at different operating temperatures. Therefore, the modules were operated in a temperature and humidity controlled cold box. The measurement procedure included a mechanical stress test by thermal cycling (10 cycles between $+17^{\circ} \mathrm{C}$ and $-25^{\circ} \mathrm{C}$ with a gradient of approximately $4.5 \frac{\mathrm{K}}{\mathrm{min}}$ ) and electrical tests before and after cycling to check for eventual degradations. A picture of the test procedure is shown in Fig. 3. During this procedure, the thresholds of the PUCs of the ROCs were unified to ensure a uniform detector response for all pixels. For the module grading, the leakage current of the sensor at different operating temperatures, the number of defective pixel cells as well as the temperature dependencies of the observables were considered [8].

\subsection{X-ray Calibration and High-Rate Test}

The modules were illuminated by monochromatic X-rays with energies up to $35 \mathrm{keV}$ as well as by non-monochromatic X-rays with rates up to $200 \frac{\mathrm{MHz}}{\mathrm{cm}^{2}}$. An easy test on the bump bonding quality can be performed by illuminating the module and looking for non-firing pixels. To calibrate the modules, monochromatic X-rays at different energies ( $\mathrm{K}_{\alpha}$-transition energies of $\mathrm{Zn}, \mathrm{Zr}, \mathrm{Mo}, \mathrm{Ag}$ ) were used. The measured signal heights and the corresponding $\mathrm{K}_{\alpha}$-transition energies can be fitted 

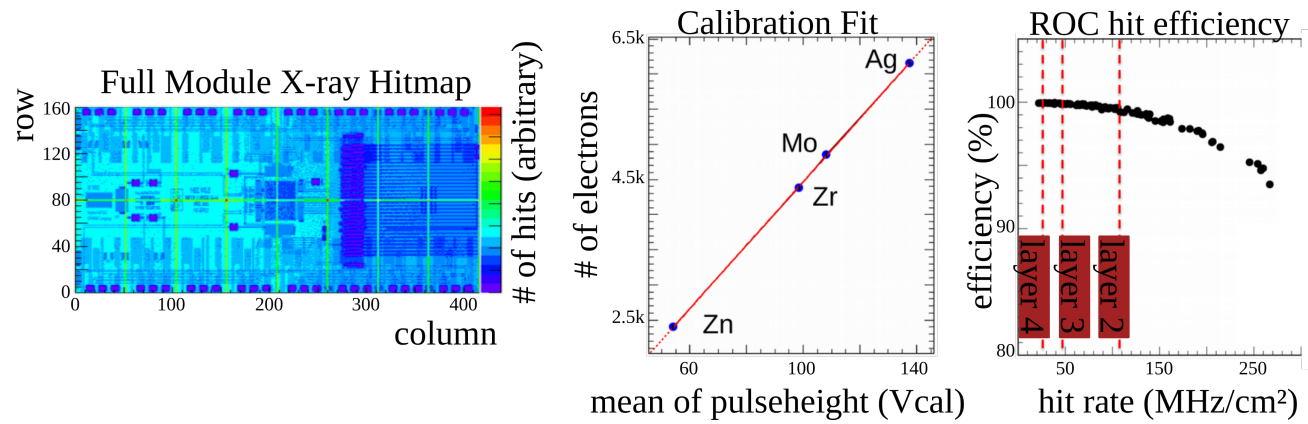

Figure 4: The figure shows a typical X-ray hitmap of a KIT/Aachen BPIX module (left), as well as a calibration fit for different X-ray target materials (center) and the ROC hit efficiency as a function of the illumination rate [8].

by linear regression to calibrate the module. With direct non-monochromatic illumination of the module, the rate capabilities of the modules can be tested. For this, test signals are injected into the PUCs while the whole module is illuminated. In case of an overload of the readout of the ROC by the high rate of firing pixels, the test signals cannot be read out, which results in a lowered hit efficiency. Figure 4 shows typical results of these procedures.

\subsection{Protection Cap and Reception Test}

As a final step, all modules were sent to ETH Zürich and equipped with a polyimide cap to protect the wire bonds during detector integration and operation. A quick optical inspection and basic electrical test was performed to sort out modules that were damaged during transport or cap gluing. In these final steps, less than $1 \%$ were damaged.

\section{Production Yields and current Production Status}

Within the 12 months of module production, the KIT/Aachen production line produced and tested 409 BPIX modules. The final grading for these modules was calculated as a combination of the thermal cycling, electrical and X-ray qualification tests. For the KIT/Aachen production line $34.2 \%$ of the module were graded as Grade A, $49.6 \%$ as Grade B and $16.1 \%$ as Grade C [8]. The main reason for the large percentage of Grade B modules was the leakage current not showing the expected temperature dependence (see sec. 2.3). Collaboration-wide, more than 1200 BPIX detector grade modules and more than 700 FPIX detector modules have been built so far and are ready for detector integration [9].

\section{References}

[1] CMS Collaboration, CMS Technical Design Report for the Pixel Detector Upgrade, PH-EP-Tech-Note-2013-001, 2013.

[2] B. Freund, Module Production and Qualification for the Phase I Upgrade of the CMS Pixel Detector, PoS(EPS-HEP2015)242, 2015. 
[3] B. Hiti, Probe Station for Functionality Tests of Bare Modules for the Phase I Upgrade of the CMS Pixel Detector, Karlsruhe Institute of Technology, Master Thesis, IEKP-KA/2015-13, 2015.

[4] A. Huffman et al., Fine-pitch wafer bumping and assembly for high density detector systems, Nuclear Science Symposium Conference Record 5, 2003.

[5] F. Colombo, Packaging and assembly technologies for the pixel detector upgrade and measurement of $\tau \tau$ final states with the CMS experiment at the LHC, Karlsruhe Institute of Technology, PHD Thesis, IEKP-KA/2016-07, 2016.

[6] S. Kudella, Gold-stud bump bonding interconnection technology for the CMS experiment, Karlsruhe Institute of Technology, Master Thesis, IEKP-KA/2014-11, 2014.

[7] A. Chilingarov, Generation current temperature scaling, CERN-LHCC-2012-016, 2012.

[8] M. Lipinski, private communication, 2016.

[9] D. Berry, private communication, 2016. 